\title{
Effects of the Soil Moisture Content on the Superoxide Anion and Proline Contents in Soybean Leaves
}

\begin{abstract}
Background: As an important source of feed protein, soybean is involved in the processing industry, food industry and other fields. Therefore, in recent years, the demand for soybean has increased and soybean planting areas have also increased. However, frequent droughts have a serious impact on soybean yield.

Methods: During the flowering period, the soybean plants were subjected to drought treatments of different degrees (0-7 days without water). The superoxide anion and proline contents in the leaves were determined. Then, fitting curves were drawn between the soil moisture content and the superoxide anion and proline contents.

Result: The effects of different soil moisture contents on the superoxide anion and proline contents in soybean leaves and the correlation between these contents were analyzed. According to the fitting curves, with a decrease in the volumetric water content of soil, the superoxide anion and proline contents in soybean leaves increased. The superoxide anion contents in drought-tolerant cultivars were significantly lower than those in drought-sensitive cultivars and the proline contents were significantly higher in droughttolerant cultivars than those in drought-sensitive cultivars. The superoxide anion content in soybean leaves was positively correlated with the proline content in the soil volumetric water content range of $31.5 \%$ to $14.5 \%$.
\end{abstract}

Key words: Drought, Proline, Soybean, Soil moisture content, Superoxide anion.

\section{INTRODUCTION}

Heilongjiang Province is the main grain production area in China and soybean is widely planted in Heilongjiang Province, accounting for more than $25 \%$ of the total cultivated land area (Yan et al., 2018). However, Zheng (1998) showed that approximately $50 \%$ of China's land area is arid and semiarid areas and approximately 1.333 million $\mathrm{hm}^{2}$ of land is damaged by drought every year. Liu et al., (2017), Padmaathi and Manohar Rao (2014) and Peng (2011) show that drought will affect antioxidant enzyme activity, membrane lipid peroxidation and other physiological and biochemical characteristics of plants. Wang (2019) and Zhu (2015) pointed that drought also reduce the photosynthetic capacity. And plants are vulnerable to drought stress in the growth and development stages according toresearches by Li et al. (2020) and Li et al. (2020).

The superoxide anion contents in plant leaf cells can characterize the degree of damage to cells under abiotic stress to a great extent. Under drought stress, a large number of superoxide anions will be produced in plants. Proline is the most important osmotic regulator defense system in leaves during drought events.

Lestari et al. (2019) and Wang (2019) showed that plants can increase the proline contents in their leaves to adapt to drought environments, maintain normal physiological function through osmotic regulation, Zhang et al. (2020) and Chen et al. (2013) showed that under the influence of drought stress, the proline content of wheat increased significantly and the increase rate of droughttolerant varieties were significantly higher those that of drought-sensitive varieties.
Northeast Agricultural University, Harbin, Heilongjiang Province, 150036, China.

Corresponding Author: Shoukun Dong, Northeast Agricultural University, Harbin, Heilongjiang Province, 150036, China.

Email: shoukundong@163.com

How to cite this article: Wu, Z., Wang, X., Wang, X., Yan, C., Ma, C., Liu, L. and Dong, S. (2021). Effects of the Soil Moisture Content on the Superoxide Anion and Proline Contents in Soybean Leaves. Legume Research. DOI: 10.18805/LR-626.

Submitted: 22-04-2021 Accepted: 04-12-2021 Online: 25-12-2021

The drought-tolerant soybean variety $\mathrm{HN} 44$ and the drought-sensitive variety HN65 were selected for this experiment. The superoxide anion and proline contents were determined under different soil moisture contents to explore the relationship between the superoxide anion and proline contents under drought stress and provide theoretical support for the drought-resistance mechanism of soybean.

\section{MATERIALS AND METHODS}

In this experiment, the soybean varieties Heinong 44 and Heinong 65 were used; Heinong 44 is drought tolerant and Heinong 65 is drought-sensitive according to Wang et al. (2012).

The experiment was carried out in a rainproof shed at Northeast Agricultural University. Plastic buckets with a height of $33 \mathrm{~cm}$ were used in the experiment. Three holes with diameters of $1 \mathrm{~cm}$ were drilled at the bottom of each bucket. The soybean plants were treated as follows: a suitable soil moisture was maintained before the $\mathrm{R} 1$ period 
Effects of the Soil Moisture Content on the Superoxide Anion and Proline Contents in Soybean Leaves

Table 1: Base fertility of tested phaeozem.

\begin{tabular}{|c|c|c|c|c|c|c|c|}
\hline Total & Total & Total & Nitratc & $\mathrm{NH}_{4}^{+}-$ & Available & Available & Organic \\
\hline $\begin{array}{l}\text { Nitrogen } \\
\mathrm{g} / \mathrm{kg}\end{array}$ & $\begin{array}{c}\text { Phosphorus } \\
\mathrm{g} / \mathrm{kg}\end{array}$ & $\begin{array}{l}\text { Potassium } \\
\mathrm{g} / \mathrm{kg}\end{array}$ & $\begin{array}{c}\text { Nitrogen } \\
\mathrm{mg} / \mathrm{kg}\end{array}$ & $\begin{array}{c}\mathrm{N} \\
\mathrm{mg} / \mathrm{kg}\end{array}$ & $\begin{array}{c}\text { Potassium } \\
\mathrm{mg} / \mathrm{kg}\end{array}$ & $\begin{array}{c}\text { Phosphorus } \\
\mathrm{mg} / \mathrm{kg}\end{array}$ & $\begin{array}{l}\text { Matter } \\
\mathrm{g} / \mathrm{kg}\end{array}$ \\
\hline 1.44 & 0.65 & 17.60 & 45.21 & 17.31 & 160.12 & 22.43 & 25.56 \\
\hline
\end{tabular}

and water supply of the first group was cut off for 7 days starting from the R1 period; $1,2,3,4,5$ and 6 days after the water supply of the first group was cut off, that of other six groups were stopped; the eighth group was the control group (CK) and the appropriate water was kept all the time for this group. Soil volumetric water content was used as the standard in the test. In this experiment, the field capacity was $42.8 \%$. According to the field moisture capacity, the drought grades were as follows: mild drought was $50-60 \%$ of the field capacity; moderate drought was $40-50 \%$ and severe drought was $30-40 \%$ of the field capacity.

\section{Index determination}

The proline content determination was conducted with the acid ninhydrin methodThe superoxide anion contents were determined according to the kit instructions (Suzhou Keming Biotechnology Co., Ltd.).

\section{Data statistics}

The experimental data were sorted and analyzed by Excel. The fitting curve were obtained by using the exponential curve fitting method in Origin software.

\section{Soil fertility and types}

The soil type is phaeozem and the soil basic fertility is as follow (Table 1).

\section{RESULTS AND DISCUSSION}

The soil moisture content, superoxide anion contents and proline contents of HN44 and HN65 under different treatments were shown in Fig 1, 2 and 3 respectively.

\section{Relationship between soil moisture content and superoxide anion content}

According to the changes observed in the superoxide anion content under different soil moisture contents, with the help of Origin software, an equation was fitted by using the exponential ( $E$ index) curve fitting method (Fig 4). The fitting equation of $\mathrm{HN} 44$ is $\mathrm{y}=0.50+1.89 \times 10^{8} \mathrm{e}^{-1.21 x}$, with $\mathrm{R}^{2}=0.962$; the fitting equation of $\mathrm{HN} 65$ is $\mathrm{y}=0.58+2.19 \times 10^{9} \mathrm{e}^{-1.36 \mathrm{x}}$, with $R^{2}=0.992$. In these equations, $y$ is the superoxide anion content and $\mathrm{x}$ is the soil moisture content. The absolute value of the change rate (denote as $|r|$ ) can reflect the variation trend of the superoxide anion content with soil moisture content. According to the above formula, the change rate of the superoxide anion content was obtained by taking the derivative of the fitting curves of the two varieties.

When $|r|$ was greater than 0 and less than 0.1 , the soil moisture content of $\mathrm{HN} 44$ was $31.5 \%-19.66 \%$, that of $\mathrm{HN} 65$ was $31.5 \%-19.41 \%$ and the superoxide anion content of

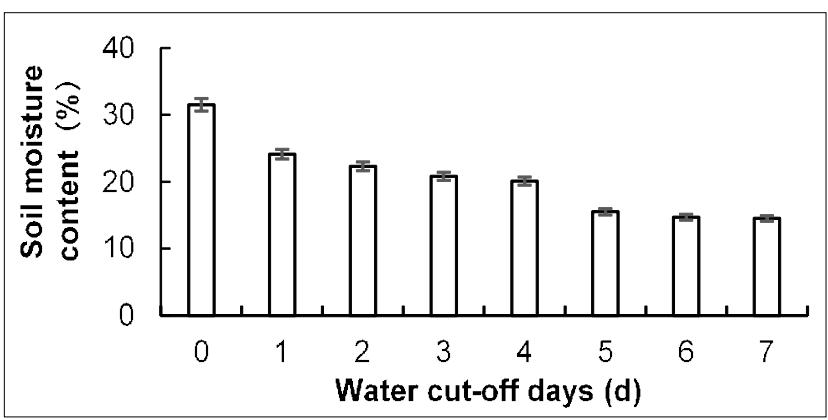

Fig 1: Soil moisture content measured on different water cutoff days.

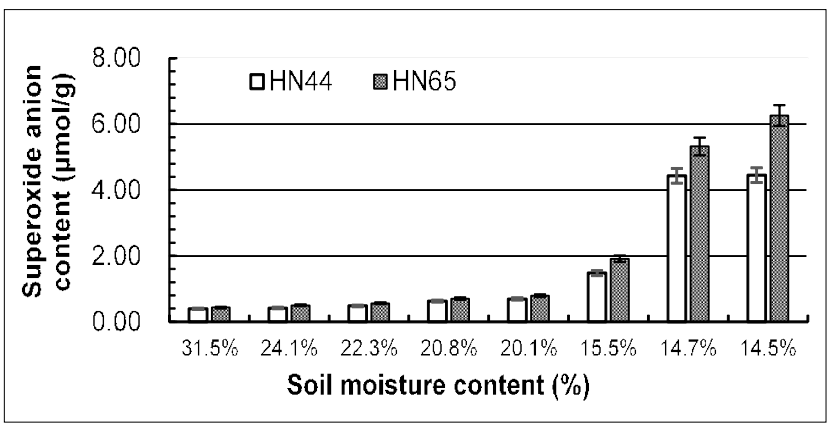

Fig 2: Effect of different soil moisture contents on the superoxide anion content of HN44 and HN65.

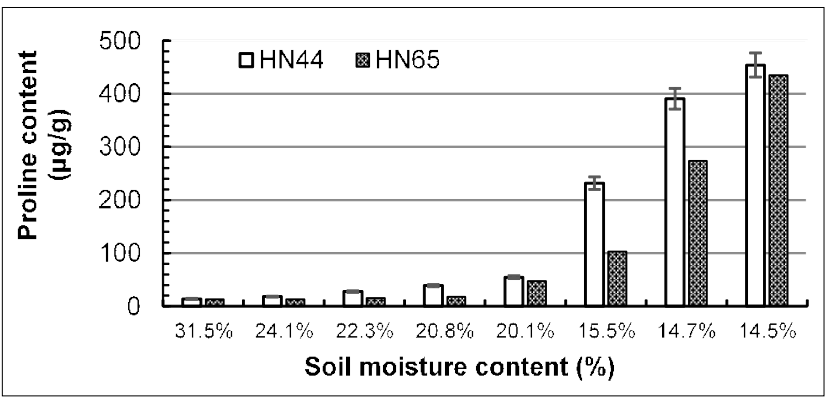

Fig 3: Effect of different soil moisture contents on the proline content of HN44 and HN65.

soybean leaves had no significant change When $|r|$ was greater than 0.1 and less than 1 , the soil moisture content of $\mathrm{HN} 44$ was $19.66 \%-17.77 \%$, that of HN65was $19.40 \%$ $17.71 \%$ and the superoxide anion content in soybean leaves increased slowly. When $|r|$ was greater than 1 , the soil moisture contents of HN44 and HN65 were $17.77 \%-14.5 \%$ and $17.70 \%-14.5 \%$, respectively and the superoxide anions contents of soybean leaves increased rapidly. The variation trends of the superoxide anion contents were similar between the two varieties. This is consistent with the research of Gao (2007), Mo (2007) and Ren (2017), etc. 
with the decrease of soil moisture content, the content of superoxide anion in leaves is increasing.

\section{Relationship between soil moisture content and proline content}

According to the changes observed in the proline contents under different soil moisture contents, an equation was fitted (Fig 5). The fitting equation of HN44 is $\mathrm{y}=27.12+1.20 \times 107 \mathrm{e}^{-0.71 \mathrm{x}}$, with $R^{2}=0.996$; the fitting equation of $\mathrm{HN} 65$ is $\mathrm{y}=23.54+4.28 \times 10^{14} \mathrm{e}^{-1.91 x}$, with $\mathrm{R}^{2}=0.984$. In these equations, $y$ is the content of proline and $x$ is the soil moisture content.

According to the above formula, the change rate of the proline content was obtained by taking the derivative of the fitting curves of the two varieties. When the $|r|$ of the proline content was greater than 0 and less than 0.1 , the soil moisture content of $\mathrm{HN} 44$ was $31.5 \%-25.73 \%$, that of $\mathrm{HN} 65$ was $31.5 \%-19.18 \%$ and the proline content of soybean leaves did not increase significantly. When $|r|$ was greater than 0.1 and less than 1 , the soil moisture content of $\mathrm{HN} 44$ was $25.73 \%-22.48 \%$, that of HN65 was $19.18 \%-17.99 \%$ and the proline content of the two soybean varieties increased slowly. When $|r|$ was greater than 1, the soil moisture content of $\mathrm{HN} 44$ was $22.48 \%-14.5 \%$, that of $\mathrm{HN} 65$ was $17.96 \%$ $14.5 \%$ and the proline content in soybean leaves increased rapidly. These results showed that drought-tolerant varieties could obviously respond to mild and moderate drought and synthesize proline earlier than drought-sensitive varieties to alleviate the effect of drought, while sensitive varieties could synthesize proline in large quantities under severe drought and their regulatory abilities were obviously weaker than those of drought-tolerant varieties.

The results showed that drought-tolerant varieties had a strong ability to accumulate osmotic adjustment substances; these substances can respond to drought earlier and reduce the damage caused by drought stress. In this experiment, the response times of the proline contents of the studied varieties with different drought tolerances to soil water content differed from the results of Sun et al. (2005) and Cai et al. (2008).

\section{Correlation analysis of the superoxide anion and prolines in soybean leaves}

A correlation analysis was conducted between the superoxide anion and proline contents in the two studied soybean varieties (Fig 6) and it can be seen that the superoxide anion and proline contents were significantly positively correlated.

The fitting equation of HN44 is $\mathrm{y}=99.36 \mathrm{x}-7.38$ and HN65 is $y=65.34 x-19.64$. The fitting correlation is preferably. The abscissa is the superoxide anion, content, the ordinate is the proline content. The data showed that there was a positive correlation between proline content and superoxide anion content in both cultivars, but the slope of HN44 was much higher than HN65, indicating that with the increase of superoxide anion content, the increase of proline content in drought-tolerant cultivars was significantly higher than that

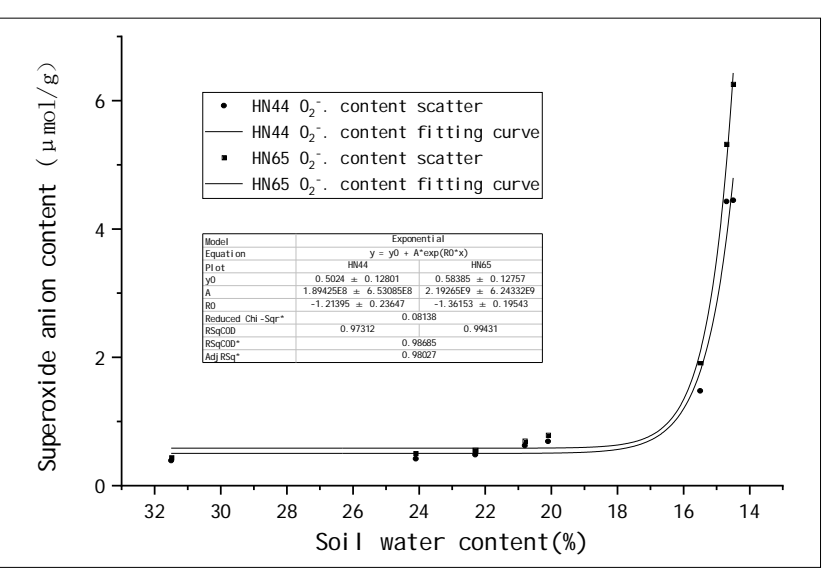

Fig 4: Fitting curve between the soil moisture content and superoxide anion contents of HN44 and HN65.

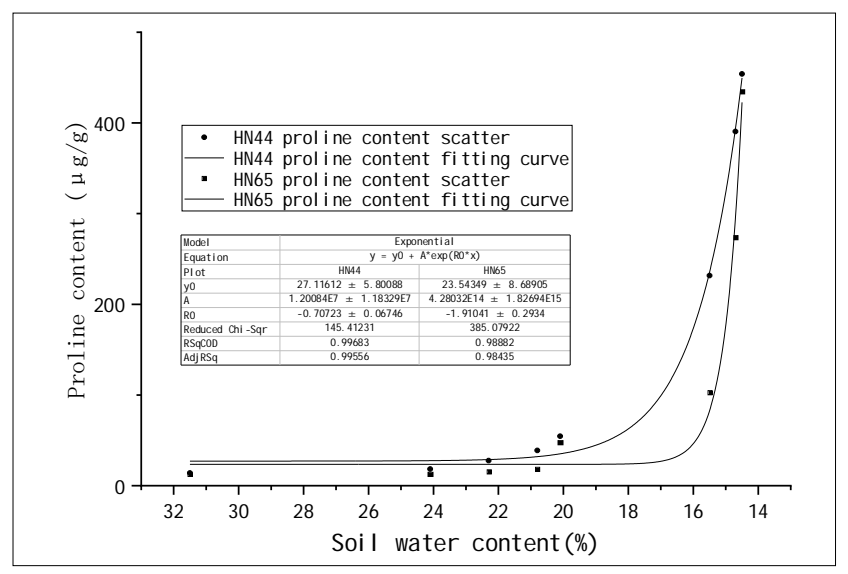

Fig 5: Fitting curve between the soil moisture content and proline contents of HN44 and HN65.

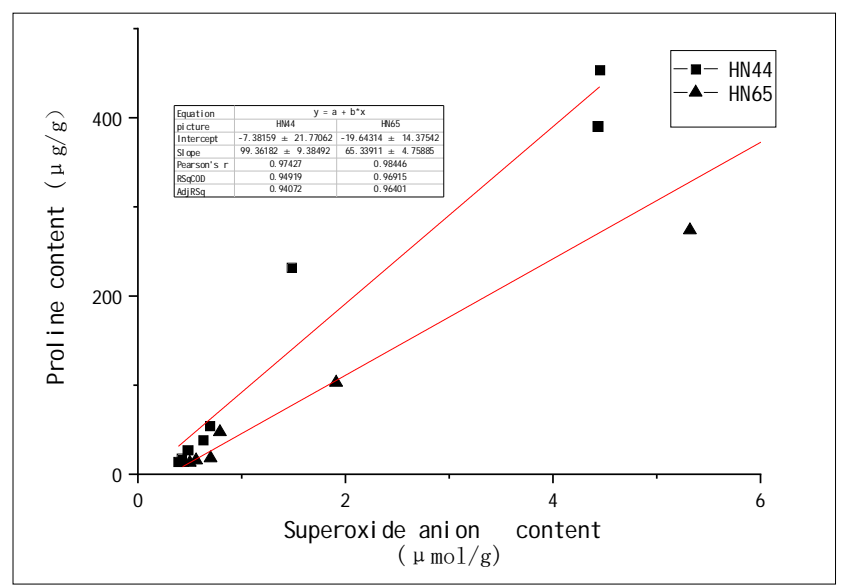

Fig 6: Correlation analysis of the superoxide anion and proline contents of HN44.

in sensitive cultivars. The results showed that proline played an important role in regulating superoxide anion content and alleviated the damage of superoxide anion on soybean under drought conditions. 


\section{CONCLUSION}

In summary, under drought stress, the superoxide anion and proline contents in soybean leaves increased to varying degrees with decreasing soil moisture contents and certain differences were observed in the responses of different varieties to drought stress. The superoxide anion contents in sensitive varieties were higher than those in droughttolerant varieties. The proline contents of drought-tolerant varieties were higher than those of sensitive varieties. Drought-tolerant varieties responded to drought earlier than drought-sensitive varieties. The correlation between the superoxide anion and proline contents in soybean leaves was significant.

\section{Funding}

This work was supported by Natural Foundation of Heilongjiang Province of China(LH2021C023).

\section{REFERENCES}

Cai, K.Z., Wu, X.Z., Luo, S.M. (2008). Effects of water stress on osmolytes at different growth stages in rice leaves and roots. Chinese Journal of Plant Ecology. 02: 491-500.

Chen, J., Gao, G., Fang, X., Wang, W., Wang, X. (2013). Analysis of seeding protein and proline content under drought stress in wheat. Journal of Anhui Agri. Sci. 41(26): 1057710578.

Gao, Y., Han, Y., Du, J.D., Zheng, D.F., Liu, J.W. (2007). Effect of drought stress on the activity of soybean enzyme. Journal of Heilongjiang Bayi Agricultural University. 04: 13-16.

Lestari, M.W., Arfarita, N., Sharma, A. and Purkait, B. (2019). Tolerance mechanisms of Indonesian plant varieties of yardlong beans (Vigna unguiculata sub sp. sesquipedalis) against drought stress. Indian J. Agric. Res. 53(2): 223227.

Li, S., Sun, X., Wang, C., Cao, Y., Wang, W., Song, S. (2020). Soybean response to drought stress under different grafting patterns. Chinese Journal of Oil Crop Sciences. 42(04): 632-639

Li, X., Ma, S., Li, Y., Yu, H., Xu, L., Chen, F., Zhang, H., Zhai, M. (2020). Effects of soil water stress on emergence and seedling growth of spring soybean. Chinese Journal of Ecology. 39(08): 2602-2609.

Liu, J., Wang, X., Bi, Y. (2017). Effects of drought stress on physiological and biochemical characteristics of soybean hypocotyls. Journal of Anhui Agricultural University. 44(02): 316-320.
Mo, H., Zhai, X. (2007). Effects of drought stress on protective enzymes activities and membrane lipid peroxidation in leaves of soybean seedlings. Hubei Agricultural Sciences. 01: 45-48.

Padmavathi, T.A.V. and Manohar Rao, D. (2014). Oxidative stress damage and antioxidant activity in 4 cultivars of peanut (Arachis hypogaea L.) under drought stress. Trends in Biosciences. 7(23): 3802-3810.

Peng, J. (2011). Effects of drought on physiological processes in soybean plants. Scientific and Technological Innovation. 32: 262.

Ren, Y., Liu, S., Qi, W., Luo, C., Gong, B., Ou, L. (2017). Changes of super oxygen anion in mulberry varieties under water stress. Guihaia. 37(09): 1122-1129:

Sun, Y., Dongfang, Y., Guo, X., Liu, Y., Yang, X., Ji, Z. (2005). Physiological characteristics and yield components in betaine aldehyde dehydrogenase transgenic rice after flowering under drought stress. Agricultural Research in the Arid Areas. 05: 108-113.

Wang, H. (2019). Effects of different functional pesticides on physiological characteristics and tuber quality of potato under drought conditions. Gansu Agricultural University.

Wang, L.B., Liu, L.J., Pei, Y.F., et al. (2012). Drought resistance identification of soybean germplasm resources at bud stage. Northeast Agric. Univ. 43(1): 36-43.

Wang, M. (2019). Photosynthetic Characteristics and Yield under Drought Stress and Evaluation of Drought Tolerance of Soybean Varieties. Shenyang Agricultural University.

Yan, C., Song, S., Wang, W., Sun, X., Wang, C., Cao, Y., Zhang, L., Li, S. (2018). Effects of different rainfall conditions on the root system of different drought-tolerant soybean. Soybean Science. 37(2): 209-214.

Zhang, P., Zhang, H., Liu, J., Huan, X., Wang, Q., Li, L., Liu, Y., Qin, P. (2020). Effects of drought and re-watering treatment on physiological and biochemical indexes of wheat cultivars/lines with different drought resistance at seedling stage. Acta Agriculturae Boreali-occidentalis Sinica. 29(12): 1-8.

Zheng, J., Huang, J. (1998). An estimation of grain loss caused by natural disasters in China, 1950-1990. Acta Geographica Sinica. 06: 23-32.

Zhu, Q. (2015). Effects of Different Drip Irrigation Treatments on Root Morphology, Photosynthesis Speciality, Flower and Pod Formation of Xindadou 27. Xinjiang Agricultural University. 